\title{
Association Between Focal Segmental Glomerulosclerosis and Cross-Fused Renalectopia: A New Cause of Secondary Focal Segmental Glomerulosclerosis or a Casual Association?
}

\author{
Rosielly Melo Tavares ${ }^{\mathrm{a}}$, Yasmin Silva e Sousa ${ }^{\mathrm{a}}$, Ginivaldo Victor Ribeiro do Nascimento ${ }^{\mathrm{a}}$, b,
}

\begin{abstract}
Cross-fused renal ectopia is a rarely described congenital kidney anomaly. It can occur in association with ureteropelvic obstruction, vesicoureteral reflux, and multicystic dysplasia, which sometimes leads to nephrolithiasis and recurrent urinary infection. However, complications related to glomerulopathies are rare in patients with congenital kidney anomaly. Here, we report a case of cross-fused renal ectopia associated with focal segmental glomerulosclerosis (FSGS). The initial clinical findings suggested the possibility of secondary FSGS; nonetheless immunosuppression was successfully done. The case demonstrates a never-before reported association in an asymptomatic patient.
\end{abstract}

Keywords: Cross-fused renal; FSGS, Glomerulosclerosis; Nephrotic syndrome; Renal ectopia; Secondary FSGS; Kidney anomaly; Renal fusion

\section{Introduction}

Cross renal ectopia is a rare congenital anomaly. It has a reported frequency of 1 case per 700 - 1,000 births, and usually (85-90\%) occurs with fusion [1]. Patients with cross renal ectopia may present with ureteropelvic obstruction, vesicoureteral reflux, and multicystic dysplasia, as well as nephrolithiasis and recurrent urinary tract infections [1].

There are only a small number of cases of congenital kidney anomalies in association with glomerulophaties in the literature. Some patients remain asymptomatic until the fourth or fifth decade of life, and 20-30\% of cases are only discovered as an accidental finding in routine exams $[1,2]$. Here, we report a case of cross-fused ectopic kidneys presenting with focal seg-

Manuscript accepted for publication March 30, 2015

${ }^{\text {aF }}$ aculdade Integral Diferencial, DeVry, Brazil

bUniversidade Estadual do Piaui, Brazil

${ }^{\mathrm{c} C o r r e s p o n d i n g ~ A u t h o r: ~ G i n i v a l d o ~ N a s c i m e n t o, ~ R . ~ V i s c o n d e ~ d a ~ P a r n a i b a, ~}$ 2315, ap 202, Horto Florestal, Teresina, PI, Brazil.

Email: gvictor.rn@uol.com.br mental glomerulosclerosis (FSGS), a combination that has not been described previously in the literature.

\section{Case Report}

A 40-year-old male patient came to the nephrologist for a routine evaluation. He had previously identified cross-fused renal ectopia, but was asymptomatic. Blood tests showed creatinine (SCr) $1.8 \mathrm{mg} / \mathrm{dL}$, blood urea nitrogen (BUN) $66 \mathrm{mg} / \mathrm{dL}$ and serum albumin $4.1 \mathrm{mg} / \mathrm{dL}$. Urinalysis revealed proteinuria $(++/ 4+)$ and hematuria (10 - 15 red blood cells/field), and 24-h proteinuria was $1.9 \mathrm{~g} /$ day. A blood lipid panel demonstrated a high total cholesterol (286 mg/dL) and high triglyceride level $(278 \mathrm{mg} / \mathrm{dL})$. The autoimmune workup was negative, serology tests for HIV and hepatitis were non-reactive, and the patient had normal complement levels.

A kidney biopsy was performed after assessment of safety and revealed seven glomeruli, four of which had global sclerosis and two of which had segmental sclerosis, with capillary occlusion and a corresponding increase in the number of mesangial cells with rare podocyte hypertrophy. Tubules with focal points of atrophy and thickening of the basal membrane were observed. There was $70 \%$ focal interstitial fibrosis with preserved vessels. Immunofluorescence microscopy demonstrated that the biopsied specimen was positive for C3. Based on these observations, a diagnosis of FSGS with diffuse interstitial fibrosis was made.

An initial treatment of enalapril, spironolactone, and prednisone $(1 \mathrm{mg} / \mathrm{kg} /$ day $)$ was administered for 16 weeks. The renal function deficit persisted with proteinuria $(2.5 \mathrm{~g} /$ day $)$. Therefore, we decided to replace the corticosteroid with $2 \mathrm{mg} / \mathrm{kg} /$ day of cyclophosphamide. After 2 months, an improvement in renal function and a reduction in proteinuria were documented. A follow-up blood test revealed changes in $\mathrm{SCr}(1.4 \mathrm{mg} / \mathrm{dL})$ and BUN $(100 \mathrm{mg} / \mathrm{dL})$, and a creatinine clearance $(\mathrm{CrCl})$ rate of 42 $\mathrm{mL} / \mathrm{min}$. His 24-h proteinuria was brought down to $280 \mathrm{mg}$. After 1 year, the patient had maintained renal function $\mathrm{CrCl}$ rate of $40 \mathrm{~mL} / \mathrm{min}$ and proteinuria at the level of $220 \mathrm{mg} /$ day.

\section{Discussion}

Congenital renal/ureteral abnormalities, including renal hypo-

Articles () The authors | Journal compilation (C) World J Nephrol Urol and Elmer Press Inc ${ }^{\mathrm{TM}}$ | www.wjnu.elmerpress.com

This is an open-access article distributed under the terms of the Creative Commons Attribution License, which permits unrestricted use, distribution, and reproduction in any medium, provided the original work is properly cited 
plasia, horseshoe kidney, simple or crossed ectopic kidneys, rotational defects and renal agenesis, occur in 3-4\% of newborns [1-3]. Clinical complications of these abnormalities consist of urinary infection, kidney stones, abdominal mass or pain, dysuria, hematuria, and oliguria. There may be anatomical manifestations, such as hydronephrosis in the ureteropelvic junction or vesicoureteral reflux $[1,2]$.

Although there are numerous descriptions of clinical complications in the literature, there are few reported cases of renal anomalies involving glomerulopathies [4]. A search in the MEDLINE and PubMed databases did not reveal any descriptions of cross-fused renal ectopia with glomerulopathy. We found only four publications describing an association between renal anomalies (horseshoe kidneys in all cases) and glomerulopathies [4-7]. FSGS was the glomerulopathy present in two of these cases, and treatment with immunosuppressant drugs did not improve proteinuria in those two cases, with levels remaining in the range of $1-2.5 \mathrm{~g}$ [4-7].

In one report, Abson et al described the case of a 52-yearold patient with peripheral edema for 6 months. The patient had proteinuria (7.7 - $14.4 \mathrm{~g} /$ day $)$, low serum albumin $(2.4 \mathrm{~g} / \mathrm{dL})$, high $\mathrm{SCr}(1.5 \mathrm{mg} / \mathrm{dL})$, and a $\mathrm{CrCl}$ of $70 \mathrm{~mL} / \mathrm{min}$. Complementary investigation showed horseshoe kidneys with FSGS. After 5 months, the $\mathrm{SCr}$ had risen to $1.7 \mathrm{mg} / \mathrm{dL}$ and $\mathrm{CrCl}$ had fallen to $53 \mathrm{~mL} / \mathrm{min}$. The patient was then started on $100 \mathrm{mg}$ cyclosporine. They described resolution of the nephrotic pathology over the first 11 months, after which the patient presented with proteinuria levels of $1-2.5 \mathrm{~g} /$ day without any relapses [5].

In the second case, Chen and Ko reported the case of 20-year-old man, admitted with repeated instances of swelling of his glans penis. A urinalysis demonstrated proteinuria (300 mg/dL), hematuria (15 - 20 red blood cells/field), and SCr and albumin levels of $0.9 \mathrm{mg} / \mathrm{dL}$ and $2.3 \mathrm{mg} / \mathrm{dL}$, respectively. Horseshoe kidneys were observed in a tomography exam, and an open kidney biopsy confirmed membranous glomerulonephritis [6].

In the third case, Fujimoto et al described the case of a 48-year-old woman with proteinuria discovered during a routine annual exam, associated with persistent hypocomplementemia. A urinalysis demonstrated proteinuria $(2+/ 4+)$ without hematuria, $\mathrm{SCr} 0.7 \mathrm{mg} / \mathrm{dL}$ and proteinuria $600 \mathrm{mg} /$ day. She had normal blood pressure and a $\mathrm{CrCl}$ of $82 \mathrm{~mL} / \mathrm{min}$, normal C3 $(89 \mathrm{mg} / \mathrm{dL})$ and low C4 $(10 \mathrm{mg} / \mathrm{dL})$. An abdominal ultrasound and tomography showed horseshoe kidneys, and a percutaneous biopsy revealed global and segmental sclerosis [7].

In the fourth report, Kavukcu et al described the case of an 18-year-old female patient submitted to evaluation of proteinuria and hematuria. She presented with a runny nose, fever, and cough, accompanied by peripheral edema. Urinalysis was 8 - 10 erythrocytes per field, $\mathrm{SCr}$ was $0.8 \mathrm{mg} / \mathrm{dL}$, albumin was $2 \mathrm{~g} / \mathrm{dL}$ and proteinuria was $1,200 \mathrm{mg} /$ day. A renal ultrasound showed evidence of horseshoe kidneys. A kidney biopsy revealed diffuse mesangioproliferative glomerulonephritis. An initial treatment with prednisolone improved the clinical signs and symptoms, although she had two episodes of massive proteinuria during follow-up. The first relapse was treated with prednisolone and the second with a combination of cyclophosphamide and prednisone [4].
It is not possible to correlate the occurrence of kidney anomalies with glomerulophaties; it can occur by chance without a causal relationship as described by Abson et al and Kavukcu et al [4, 5]. However, different from primary FSGS, which is related to minimal changes and profuse proteinuria, secondary FSGS can be associated to pre-existing conditions and occur in various forms. Moreover, these patients are more likely to have mild foot process effacement and to present with subnephrotic-range proteinuria and normal serum albumin $[8$, 9].

The term FSGS is used to describe a histopathological glomerular sclerosis lesion. It is considered to be secondary when it arises in the presence of a pre-existing pathology, including lymphoproliferative disorder, autoimmune diseases, infection, unilateral renal artery stenosis, increased growth hormone production, dense deposit disease, and lipid deposition. It also includes congenital vesicoureteral reflux and renovascular diseases [10-12].

Secondary FGSG is known to result from hemodynamic adaptations mediated by intrarenal vasodilatation leading to glomerular hypertrophy and hyperfiltration [8-11]. There are no prior descriptions of this occurrence in patients with crossfusion ectopia, although this condition is known to be related to a high variability of vasculature $[2,9]$ which makes it possible that some degree of hemodynamic adaptation can occur in these cases.

The first line therapy in secondary FSGS is blockade of the renin angiotensin system and dietary sodium restriction. There is no evidence to support administration of glucocorticoid therapy in such cases $[8,9]$. Immunosuppressive therapy should be considered if there is progression to full nephrotic syndrome in patients with foot process effacement evidenced by electronic microscopy [9]. Calcineurin inhibitors can be used as an empirical therapy in some genetic forms of the disease [8].

For primary FSGS treatment should start with corticosteroids over a minimum period of 3 months. Immunosuppressant drugs (cyclophosphamide, chlorambucil, or azathioprine) are a second line of treatment for patients that are resistant to corticotherapy, with maximum remission of symptoms occurring within 6 months. Non-specific therapies (ACEIs, angiotensin II receptor blockers, and statins) are often beneficial and should be considered [12].

This present case shows a never-before described association between cross-fused renal ectopia and FSGS. The clinical evolution contributed to our determination of the type of FGSG. Despite an initial failure to respond to steroid treatment, the patient's excellent response to cyclophosphamide characterized by complete remission of proteinuria and maintenance of renal function indicates the possibility of a primary FSGS. Distinguishing between primary and secondary FSGS was somehow difficult in this case because of the initial presentation. Few clinical findings, subnephrotic-range proteinuria, and normal serum albumin associated with kidney anomaly featuring a wide variety of vascularization point to likely secondary FSGS. Although percutaneous kidney biopsy is known to be associated with technical difficulties, it was performed successfully, allowing an accurate diagnosis, adminis- 
tration of an appropriate treatment and a stable renal function for at least a year.

\section{Conflict of Interest}

The authors declare that they have no conflict of interest.

\section{References}

1. Oliveira CM, Santos DC, Gomes DM, Choukroun G, Kubrusly M. [Crossed renal ectopia with fusion: report of two cases and review of the literature]. J Bras Nefrol. 2012;34(3):283-287.

2. Glodny B, Petersen J, Hofmann KJ, Schenk C, Herwig R, Trieb T, Koppelstaetter C, et al. Kidney fusion anomalies revisited: clinical and radiological analysis of 209 cases of crossed fused ectopia and horseshoe kidney. BJU Int. 2009;103(2):224-235.

3. Maranhao CPM, Miranda CMNR, Santos CJJ, Farias LPG, Padilha IG. Congenital upper urinary tract abnormalities: new images of the same diseases. Radiol Bras. 2013;46(1):43-50.
4. Kavukcu S, Sahin B, Turkmen M, Soylu A, Lebe B, Buyukgebiz A. Hoerseshoe kidney with mesangioproliferative glomerulonephiris and goiter. Turk J Pediatr. 2003;45(4):350-352.

5. Abson C, Jones M, Palmer A, Persey M, Gabriel R. Horseshoe kidney, focal and sclerosing glomerulonephritis and primary hypothyroidism. Nephron. 1991;58(1):124.

6. Chen A, Ko WS. Horseshoe kidney and membranous glomerulonephropathy. Nephron. 1990;54(3):283-284.

7. Fujimoto S, Hirayama N, Uchida T, Iemura F, Yamamoto Y, Eto T, Washimine H, et al. Horseshoe kidney and membranous glomerulonephritis with cold activation of complement. Intern Med. 1992;31(5):625-628.

8. D'Agati VD, Kaskel FJ, Falk RJ. Focal segmental glomerulosclerosis. N Engl J Med. 2011;365(25):2398-2411.

9. Sethi S, Glassock RJ, Fervenza FC. Focal segmental glomerulosclerosis: towards a better understanding for the practicing nephrologist. Nephrol Dial Transplant. 2015;30(3):375-384.

10. Mallick N. Secondary focal glomerulosclerosis not due to HIV. Nephrol Dial Transplant. 2003;18(Suppl 6):vi64-67.

11. Ferrario F, Rastaldi MP, Pasi A. Secondary focal and segmental glomerulosclerosis. Nephrol Dial Transplant. 1999;14(Suppl 3):58-67. 